\title{
Copper Particle Effect on the Breakdown Strength of Insulating Oil at Combined AC and DC Voltage
}

\author{
You-Yuan Wang ${ }^{\dagger}$, Yuan-Long $\mathrm{Li}^{*}$, Chao Wei**, Jing Zhang* and $\mathrm{Xi} \mathrm{Li}^{*}$
}

\begin{abstract}
Converter transformer is the key equipment of high voltage direct current transmission system. The solid suspending particles originating from the process of installation and operation of converter transformer have significant influence on the insulation performance of transformer oil, especially in presence of DC component in applied voltage. Under high electric field, the particles easily lead to partial discharge and breakdown of insulating oil. This paper investigated copper particle effect on the breakdown voltage of transformer oil at combined AC and DC voltage. A simulation model with single copper particle was established to interpret the particle effect on the breakdown strength of insulating oil. The experimental and simulation results showed that the particles distort the electric field. The breakdown voltage of insulating oil contaminated with copper particle decreases with the increase of particle number, and the breakdown voltage and the logarithm of particle number approximately satisfy the linear relationship. With the increase of the DC component in applied voltage, the breakdown voltage of contaminated insulating oil decreases. The simulation results show that the particle collides with the electrode more frequently with more DC component contained in the applied voltage, which will trigger more discharge and decrease the breakdown voltage of insulating oil.
\end{abstract}

Keywords: Converter transformer, Insulating oil, Copper particle, Combined AC and DC voltage, Breakdown strength, Weibull distribution, Particle trajectory

\section{Introduction}

Converter transformer is the key component connecting the $\mathrm{AC}$ power grid and $\mathrm{DC}$ power grid in the high voltage direct current (HVDC) transmission systems, and the insulating oil of converter transformer simultaneously withstands AC voltage and DC voltage [1]. The insulating performance of transformer oil is quite sensitive to solid particles suspending in the transformer insulating oil, which originate from the mechanical vibration, aging and partial discharge of converter transformer insulation [2]. The particles in transformer insulating oil will move to the high electric field region and form impurity bridges due to the electric field force, which will reduce the breakdown strength of the insulating oil [3]. The motion of particles at combined $\mathrm{AC}$ and $\mathrm{DC}$ voltage is different from that at AC voltage, which will lead to different breakdown characteristics of contaminated insulating oil [4]. Investigation into the breakdown characteristics of transformer insulating oil contaminated with solid particles at combined $\mathrm{AC}$ and $\mathrm{DC}$ voltage is conductive to the understanding of breakdown mechanism of insulating oil used in converter

$\dagger$ Corresponding Author: Dept. of Electrical Engineering, Chongqing University, China. (lyloog@163.com)

* Dept. of Electrical Engineering, Chongqing University, China. (\{y.wang, zhangjing2012\}@cqu.edu.cn,478602519@qq.com )

** Power Equipment Condition Assessment Center, Jiangsu Electric Power Company Research Institute, China.

(15105168905@163.com)

Received: August 5, 2016; Accepted: December 6, 2016 transformer in presence of particle contamination.

The particle type, particle concentration and the external electric field are the key factors influencing the breakdown strength of insulating oil [5]. The CIGRE report [6] suggested that particle identification and counting were found to be necessary procedures of condition monitoring of converter transformers. In view of the research progress of the particle effect on the breakdown of insulating oil, focus was made on the particle types [7-9], voltage types $[10,11]$ particle movement $[12,13]$ under electric field, and partial discharge $[10,13]$ in insulating oil. Reference $[7,8]$ observed that conducting particles affect the dielectric strength much more than insulating particles, and larger particles reduce the breakdown voltage even more significantly. Reference [9] obtained insulating oil with different content of carbon particles through different numbers of lightning discharge and investigated the influence of carbon particle concentration on the breakdown voltage of insulating oil at AC voltage. Reference [10] investigated the influence of conductive particle with different particle sizes on the breakdown voltage of insulating oil at DC voltage, and it was found that larger particle has significant influence on the breakdown of insulating oil. References [12] investigated the motion of conductive particle at $\mathrm{AC}$ voltage. The $\mathrm{AC}$ breakdown strength of insulating oil contaminated with different copper particle concentrations was obtained. Reference [13] also investigated the motion of single steel particle in 
insulating oil at $\mathrm{AC}$ voltage and obtained the relationship between the PDs on particle and the motion of particle in insulating oil. Most publications investigated particle effect on the breakdown characteristics of insulating oil at individual AC voltage and individual DC voltage. Relatively few publications dealt with the influence of particle on the breakdown voltage at combined AC and DC voltage. Most publications analyzed the influence of particle on the breakdown voltage of insulating oil by means of the mass weight or concentration. However, the number of particles was seldom precisely considered, let alone the relationship between particle number and breakdown voltage at combined AC and DC voltage.

The objective of this paper is to investigate the influence of copper particle on the breakdown characteristics of insulating oil at combined AC and DC voltage and analyze the mechanism behind it with the simulation result of the copper particle movement trajectory. Firstly, insulating oil with different numbers of copper particles were prepared. Subsequently, the breakdown strength of insulating oil contaminated with copper particles at combined $\mathrm{AC}$ and $\mathrm{DC}$ voltage with the ratio of $\mathrm{AC}$ to $\mathrm{DC}$ component equal to 1 and $1 / 3$ were measured, and breakdown strength at individual $\mathrm{AC}$ and $\mathrm{DC}$ voltage were also measured for comparison. The Weibull distribution was used to analyze the breakdown strength of contaminated insulating oil. Lastly, the electric field distribution near a single copper particle in the insulating oil was calculated and the particle movement trajectory is simulated to interpret the breakdown characteristic of contaminated insulating oil at combined AC and DC voltage.

\section{Experiments}

\subsection{Experimental setup}

The experimental set up is shown in Fig. 1, which consists of a function generator, an amplifier, a voltage divider and an oscilloscope. The input signal was provided by the function generator with the voltage types of $\mathrm{AC}$, DC and combined AC and DC voltage. Reference [14]

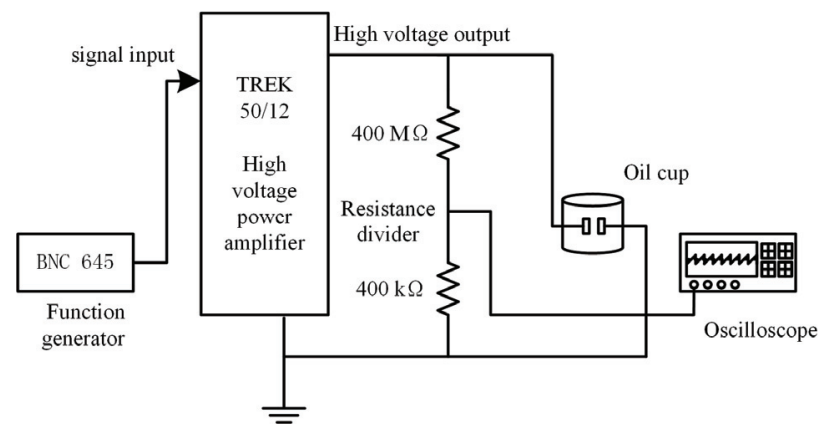

Fig. 1. Wiring diagram of insulating oil breakdown test system analyzed the voltage type that the converter transformer valve windings withstands and it found that the ratio of maximum value for $\mathrm{AC}$ and $\mathrm{DC}$ component is mainly 1 and $1 / 3$. In this paper, the combined $A C$ and $\mathrm{DC}$ voltage with the ratio of $A C$ to $D C$ equal to 1 and $1 / 3$ were adopted during the experiments. The output signal of the signal generator is amplified by the high voltage amplifier. The electrode system in the oil cup is designed according to ASTM D877 [15], as shown in Fig. 2.

\subsection{Experimental samples}

The Karamay 25\# transformer insulating oil provided by China National Petroleum Corporation was used as the insulating oil for experiments. The basic physical and chemical properties of the insulating oil are shown in Table 1. In order to eliminate water and particle effect, the transformer insulating oil was firstly filtered by a membrane with the pore size of about $0.8 \mu \mathrm{m}$. The filtered oil satisfies the "clean oil" standard [5], namely, the number of particles whose diameter larger than $5 \mu \mathrm{m}$ is less than 300 per $100 \mathrm{ml}$ in the oil sample. Then the insulating oil was degassed and dried at $80{ }^{\circ} \mathrm{C}$ for 48 hours to make sure that the water content in the insulating oil is less than $15 \mathrm{mg} / \mathrm{L}$.

The particle in insulating oil has a wide range of diameter, mainly ranging from $1 \mu \mathrm{m}$ to $100 \mu \mathrm{m}$ [5]. Copper particles are typically metallic particles found in insulating oil. Therefore, copper particles were used in this experiment to investigate the metallic particle effect on the breakdown strength of insulating oil. Their median diameter is $25 \mu \mathrm{m}$

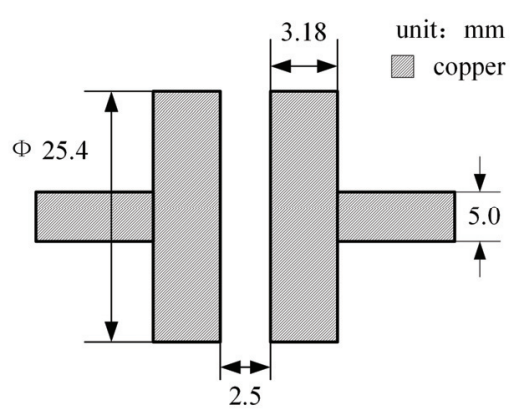

Fig. 2. The plate-plate electrode system

Table 1. Basic physical and chemical properties of Karamay 25\# transformer insulating oil

\begin{tabular}{c|c}
\hline Parameter & Mineral oil \\
\hline Appearance & Transparent \\
\hline Density $\left(20^{\circ} \mathrm{C}\right) / \mathrm{kg} \cdot \mathrm{m}^{-3}$ & $<0.895$ \\
\hline Kinematic viscosity $\left(40^{\circ} \mathrm{C}\right) / \mathrm{mm}^{2} \cdot \mathrm{s}^{-1}$ & $\leq 13.0$ \\
\hline Pour point $/{ }^{\circ} \mathrm{C}$ & $<-22$ \\
\hline Flash point $/{ }^{\circ} \mathrm{C}$ & $\geq 135$ \\
\hline Acid value $/ \mathrm{mgKOH} \cdot \mathrm{g}^{-1}$ & $\leq 0.03$ \\
\hline Interfacial tension $/ \mathrm{mN} / \mathrm{m}$ & 47 \\
\hline Dissipation factor $\left(90^{\circ} \mathrm{C}\right)$ & $0.1 \%$ \\
\hline Relative permittivity $\left(90^{\circ} \mathrm{C}\right)$ & 2.2 \\
\hline Volume resistivity $\left(90^{\circ} \mathrm{C}\right) / \Omega \cdot \mathrm{m}$ & $7 \times 10^{11}$
\end{tabular}




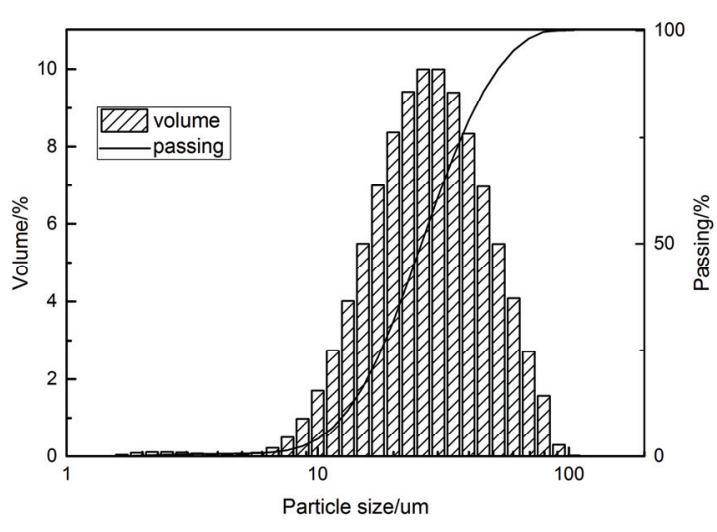

Fig. 3. Particle size distribution of copper

and the particle size distribution range is $1-100 \mu \mathrm{m}$. The median diameter is a criterion to evaluate the overall particle size distribution of particles. It means the diameter at which the cumulative size distribution percentage reaches $50 \%$. The copper particle size distribution is shown in Fig. 3.

In this experiment, insulating oil with five copper particle concentrations were prepared. $N$ was used to represent the particle number in per $100 \mathrm{ml}$ oil. $N$ was fixed at 10000, 100000, 500000, 1000000 and 5000000, symbolized as $N 1, N 2, N 3, N 4$ and $N 5$, respectively. $N$ was determined by measuring the mass weight of copper particles. The relationship between the particle number and particle mass weight is illustrated in appendix.

\subsection{Testing procedures}

The experiments of breakdown strength of contaminated oil were carried out as per ASTM D877 [15]. The increasing speed of voltage is about $2 \mathrm{kV} / \mathrm{s}$. In order to get plenty of experimental data for statistical analysis, three series of 6 breakdown voltages were measured. During the test interval, an electromagnetic stir was used to stir the oil sample to ensure the homogeneity of particle distributions in the insulating oil, and the standing time of 2 minutes between two successive breakdowns was adopted to make the particles back to state of homogeneous distribution. The breakdown voltage of insulating oil contaminated with five copper particle concentrations was measured at $\mathrm{AC}$ voltage, DC voltage and combined $\mathrm{AC}$ and $\mathrm{DC}$ voltages.

\section{Experimental Results}

\subsection{Weibull distribution analysis}

Statistical methods (parametric and non-parametric) have been used to study the breakdown voltage distribution of transformer oil [16]. The common distributions are normal distribution, Gumbel distribution and Weibull distribution. Previous study showed that Weibull distri-

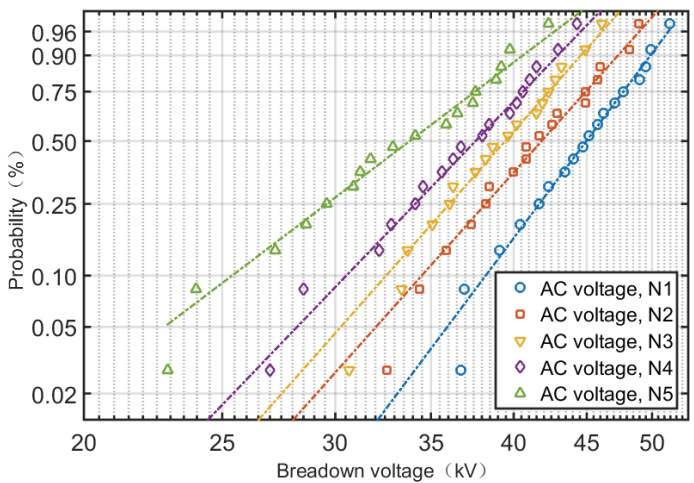

Fig. 4. Weibull probability plot of breakdown voltage of insulating oil contaminated with copper particles at AC voltage

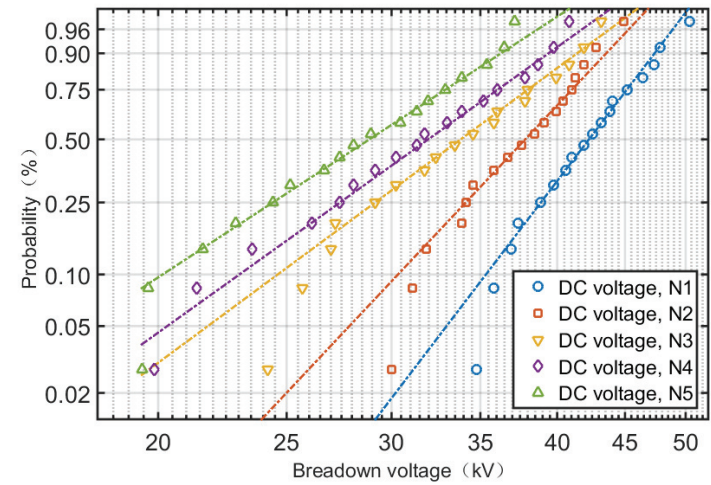

Fig. 5. Weibull probability plot of breakdown voltage of insulating oil contaminated with copper particles at DC voltage

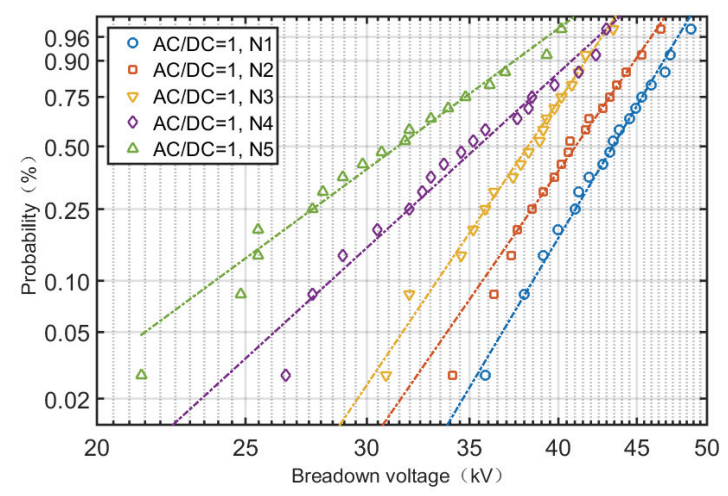

Fig. 6. Weibull probability plot of breakdown voltage of insulating oil contaminated with copper particles at $\mathrm{AC} / \mathrm{DC}=1$

bution has some advantages over other distributions to analyze breakdown voltage data of insulating oil [17]. In this paper, the two-parameter Weibull distribution model was used to analyze the breakdown voltage. The twoparameter Weibull distribution is shown in Eq. (1), where $F(u)$ is the probability of breakdown, $\alpha$ is the scale parameter and $\beta$ is the shape parameter and $u$ is breakdown 
Table 2. Characteristic parameter of Weibull distribution of breakdown voltage

\begin{tabular}{c|c|c|c|c|c|c}
\hline $\begin{array}{c}\text { Voltage } \\
\text { type }\end{array}$ & $\begin{array}{c}\text { Weibull } \\
\text { parameter }\end{array}$ & $N 1$ & $N 2$ & $N 3$ & $N 4$ & $N 5$ \\
\hline \multirow{2}{*}{$\mathrm{AC}$} & $\alpha$ & 46.39 & 43.36 & 40.85 & 38.91 & 35.64 \\
\cline { 2 - 7 } & $\beta$ & 12.44 & 10.51 & 10.89 & 9.71 & 7.37 \\
\hline \multirow{2}{*}{$\mathrm{DC}$} & $\alpha$ & 44.04 & 39.38 & 36.25 & 33.81 & 30.80 \\
\cline { 2 - 7 } & $\beta$ & 10.85 & 10.43 & 6.88 & 6.11 & 6.09 \\
\hline \multirow{2}{*}{$\mathrm{AC} / \mathrm{DC}=1$} & $\alpha$ & 44.52 & 42.22 & 39.34 & 37.16 & 33.29 \\
\cline { 2 - 7 } $\mathrm{AC} / \mathrm{DC}=1 / 3$ & $\beta$ & 15.03 & 14.22 & 14.24 & 8.32 & 6.88 \\
\cline { 2 - 7 } & $\beta$ & 44.12 & 40.64 & 37.63 & 35.28 & 31.96 \\
\hline
\end{tabular}

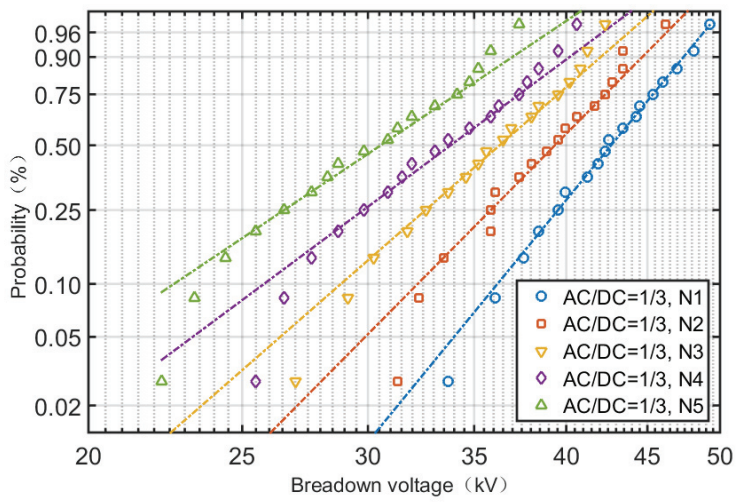

Fig. 7. Weibull probability plot of breakdown voltage of insulating oil contaminated with copper particles at $\mathrm{AC} / \mathrm{DC}=1 / 3$

voltage of insulating oil.

$$
F(u)=1-e^{-(u / \alpha)^{\beta}}
$$

Figs. 4-7 show the Weibull distribution of the breakdown voltage of insulating oil contaminated with copper particles. The Weibull distribution of breakdown voltage in Figs. 4-7 show that the breakdown voltage data of contaminated oil are distributed in both sides of the Weibull probability straight line, which indicates that the breakdown voltage data of contaminated oil almost satisfy the Weibull distribution.

The scale parameter $\alpha$ and shape parameter $\beta$ of the breakdown voltage were calculated with maximum likelihood estimation. The results are shown in Table 2. The scale parameter $\alpha$ of Weibull distribution of the breakdown voltage was adopted as the characteristic breakdown voltage.

\subsection{Fitting results}

The relationship between the copper particle concentration and the breakdown voltage was depicted in Fig. 8. A linear function was used to fit the above relationship, as shown in Table 3, where $U$ is the characteristic breakdown voltage of contaminated oil and $\lg N$ is the
Table 3. Fitting expressions of the breakdown voltage of insulating oil

\begin{tabular}{c|c}
\hline Voltage type & Fitting expression \\
\hline $\mathrm{AC}$ & $U=-3.9697 \lg N+62.7843$ \\
\hline $\mathrm{DC}$ & $U=-4.9559 \lg N+64.0097$ \\
\hline $\mathrm{AC} / \mathrm{DC}=1$ & $U=-4.1562 \lg N+62.0789$ \\
\hline $\mathrm{AC} / \mathrm{DC}=1 / 3$ & $U=-4.5420 \lg N+62.8155$ \\
\hline
\end{tabular}

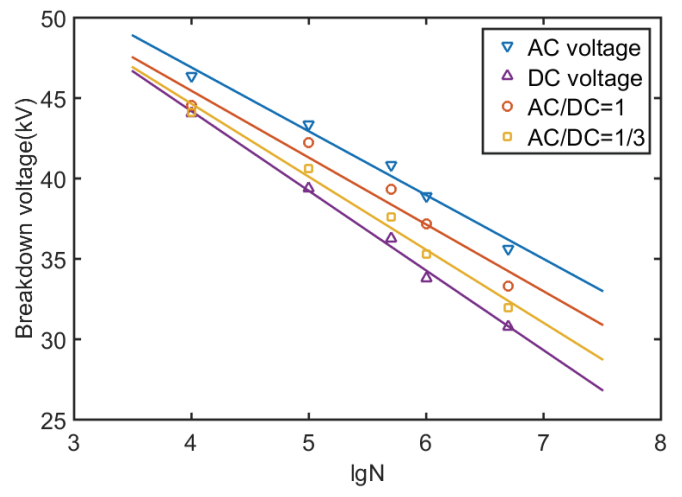

Fig. 8. Breakdown voltage of insulating oil contaminated with copper particles

logarithm of the copper particle number in per $100 \mathrm{ml}$ of insulating oil.

It can be seen from Fig. 8 and Table 3 that the breakdown voltage decreases with the increase of particle concentration. The above changing tendency demonstrates that the copper particle concentration has significant influence on the breakdown voltage of insulating oil, regardless of voltage type. The breakdown voltage reduces with the increase of the $\mathrm{DC}$ component at the same particle concentration, i.e. breakdown voltage at $\mathrm{AC}$ voltage is the largest while breakdown voltage at DC voltage is the smallest, and breakdown voltage at combined $\mathrm{AC}$ and $\mathrm{DC}$ voltage is medium.

Fig. 8 presented the fitting results of breakdown voltage of contaminated insulating oil versus particle number. The breakdown voltage reduces almost $3.5-5 \mathrm{kV}$ with tenfold increase in copper particle number. The decreasing speed of the breakdown voltage at combined AC and DC voltage is medium. The decreasing speed of the breakdown voltage at $\mathrm{AC}$ voltage is the smallest while $\mathrm{DC}$ voltage largest.

\section{Discussions}

\subsection{Simulation model}

According to the electrode system, a simulation model with single copper particle was established with the COMSOL simulation software to investigate the electric field distortion caused by the single copper particle and the copper particle trajectory, as shown in Fig. 9. The COMSOL software provides two module called "Particle 
Table 4. Material parameter

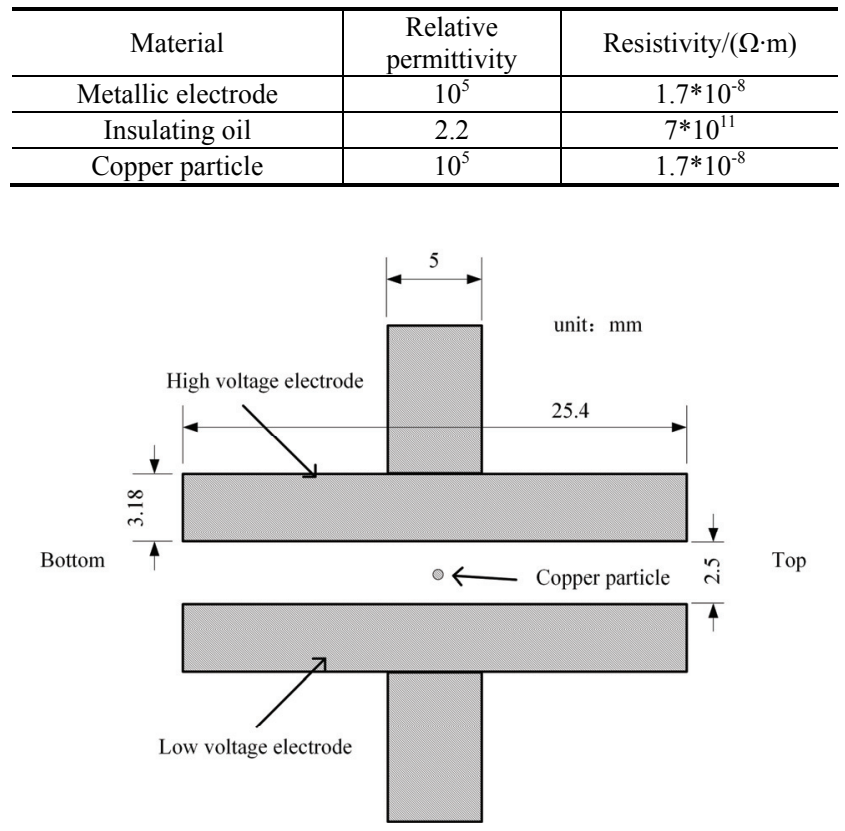

Fig. 9. COMSOL simulation model of copper particle

Tracing" and "AC/DC", which are enough to accomplish this task. The copper particle was placed in the axis of symmetry of the high voltage electrode and the low voltage electrode. The diameter of copper particle is $25 \mu \mathrm{m}$. The insulation around the electrode and copper particle is transformer insulating oil. The peak value of applied voltage on the high voltage electrode is $10 \mathrm{kV}$. The low voltage electrode is grounded. The material parameter of the simulation model is shown in Table 4.

The forces applied on the copper particle in this simulation are drag, electrostatic and gravitational forces. Reference [18] found that the conductive particle in an electric field will be charged by contacting the electrode. The charge on the copper particle is calculated by Eq. (2) [19].

$$
q=\frac{2}{3} \pi^{3} \varepsilon_{m} \varepsilon_{0}\left(\frac{d_{p}}{2}\right)^{2} E
$$

where $\varepsilon_{m}, \varepsilon_{0}, d_{p}$ and $E$ are the relative permittivity of the liquid, the permittivity of vacuum, the diameter of copper particle, and the electric field, respectively.

The trajectory of copper particle is also influenced by the gravitational force and drag force during the course of movement in the insulating oil. The gravitational force $F_{g}$ and drag force $F_{d}$ are given by Eqs. (3) and (4) respectively.

$$
F_{g}=m_{p} g \frac{\left(\rho_{p}-\rho\right)}{\rho_{p}}
$$

where $g$ is the acceleration of gravity, $m_{p}$ is the mass of the

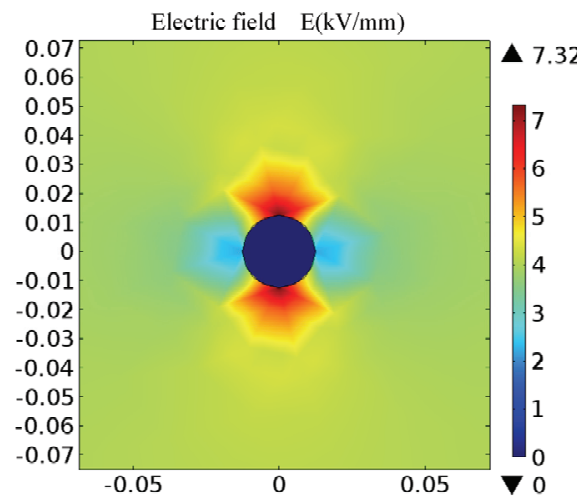

Fig. 10. The electric field distribution around copper particle at $\mathrm{AC} / \mathrm{DC}=1$

particle, $\rho_{p}$ is the mass density of copper particle, and $\rho$ is the mass density of insulating oil.

$$
F_{d}=\frac{18 \mu}{d_{p}^{2} \rho_{p}} m_{p} v
$$

where $\mu$ is the viscosity of insulating oil, $v$ is the velocity of copper particle.

\subsection{Electric field analysis}

Fig. 10 shows the simulation results of the electric field distribution around a single copper particle at combined $\mathrm{AC}$ and DC voltage with the ratio of $\mathrm{AC}$ to $\mathrm{DC}$ equal to 1. Results show that the electric field between the two electrodes is uniform except for the regions around the particle and the value is $4 \mathrm{kV} / \mathrm{mm}$. There is a distortion in the electric field around the copper particle. The maximum of the distorted electric field is about $7.32 \mathrm{kV} / \mathrm{mm}$ and the distorted area exists above and below the copper particle. The simulation results show that the introduction of copper particles will lead to the distortion of local electric field strength in the insulating oil. This phenomenon is also observed at other types of voltage. The uniformity coefficient of electric field can be calculated by Eq. (5) [20].

$$
f_{C u}=\frac{E_{\max }}{E_{\text {mean }}}=7.32 / 4=1.83
$$

where $E_{\max }$ represents the maximum electric field, and $E_{\text {mean }}$ represents the mean value of electric field.

Fig. 11 shows the electric field distribution around copper particle in one period at AC voltage, DC voltage, and combined $\mathrm{AC}$ and DC voltage. The DC component has significant influence on the electric field. As the increase of the DC component in combined AC and DC voltage, the electric field strength around copper particle will increase while the maximum value stays the same and the volatility of electric field strength will reduce. 


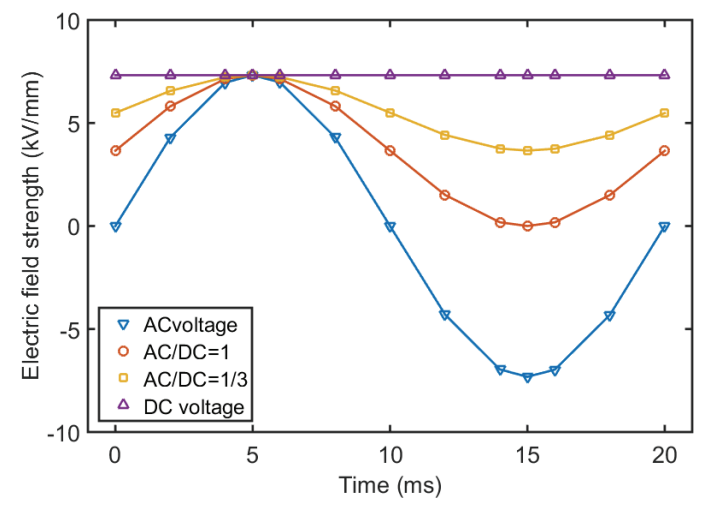

Fig. 11. The electric field distribution around copper particle

Table 5. Copper particle trajectories

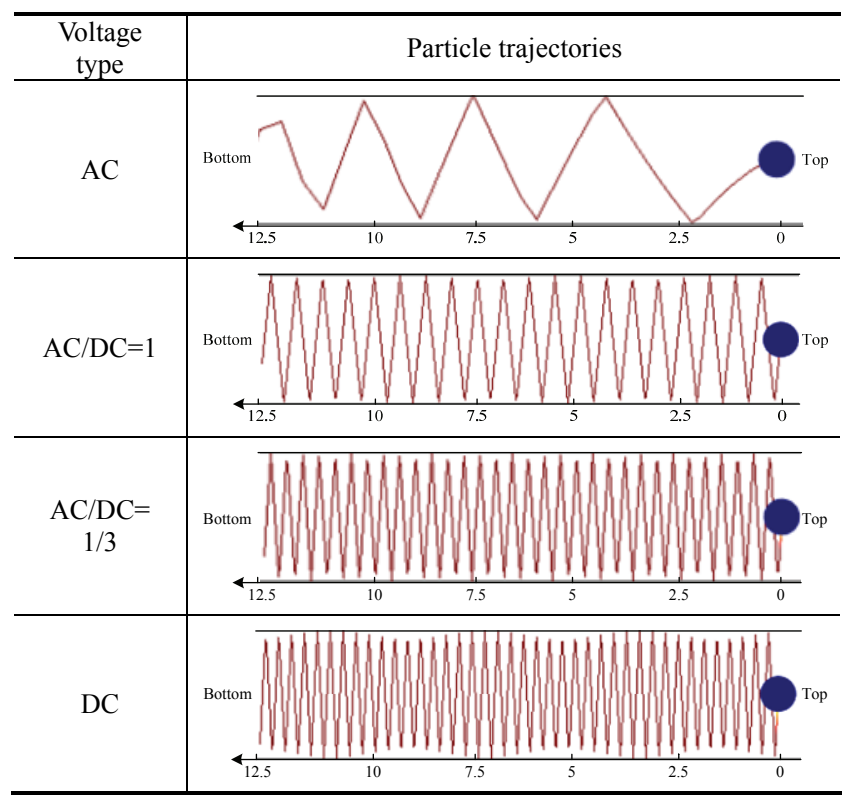

The trajectories of the copper particle between the high voltage electrode and low voltage electrode at AC voltage, DC voltage and combined AC and DC voltage are shown in Table 5. The particle trajectory of simulation result at DC voltage is similar with the experimental result obtained in [21] and [22]. The particle trajectories show the path that copper particle pass through between the plate electrodes. It can be concluded from Table 5 that the copper particle collides with the electrode more frequently at DC voltage and the numbers of collisions at combined AC and DC voltage is medium. With the increase of the DC voltage component in applied voltage, the copper particle collides with the electrode more frequently. In summary, the voltage type has significant influence on the particle movement and the DC voltage will increase the collision probability with electrode.

\subsection{Mechanism analysis}

The influence of copper particle on the breakdown voltage of insulating oil can be explained with the simulation results as follows.

The electric filed simulation results show that a certain amount of charge will be induced in the two sides of the copper particle when reaching the electrode. These charges will form an additional electric filed around the copper particle. The additional electric field and original electric field in insulating oil will cause the electric field distortion, namely, the electric field near the copper particle will obviously increase.

As the copper concentration is low, it can be considered that the breakdown of insulating oil is caused by individual particle. Reference [21] found that particles will move to high electric field and contact the electrode due to the effect of electric force. From the simulation results, it is clear that the electric field in insulating oil with the existence of a copper particle is much larger than that without a copper particle. When the particle moves to the area with larger electric field, the electric field will be distorted further. In that case, it is easier to trigger discharge. When the electric field strength exceeds the breakdown strength of insulating oil, the insulating oil breakdowns [18, 23]. Even if the electric field strength around particle surface is not large enough, the particles will move to the electrode that carries charge with the opposite polarity due to the effect of electric force. When the particle reaches the area near the electrode, the electric field strength between the particle and electrode will increase, which will cause partial breakdown and finally lead to the entire breakdown of the oil gap [12].

As the copper particle concentration of insulating oil is large, more and more particles will concentrate in electrode area with a strong electric field due to the electric force, which leads to the increase of copper particles concentration. Then the collision frequency with electrode of copper particles will increase. A large amount of copper particles movement will form a phenomenon which is similar to the impurity bridge [24].

As to the difference between the AC voltage and DC voltage, the copper particle will withstand an electric force with a constant polarity at DC voltage and combined AC and DC voltage. The particles are easier to gather in the high electric field area, including the electrode surface [25]. The copper particles are much easier to contact the two electrodes, as shown in Table 5. Therefore, the breakdown voltage of insulating oil at DC voltage, combined AC and $\mathrm{DC}$ voltage is smaller than that at $\mathrm{AC}$ voltage. This phenomenon is in accordance with the experimental results.

For converter transformers, the valve side winding withstands combined AC and DC voltages. Because the copper particle has larger influence on the breakdown voltage of insulating oil when subjected to DC voltage, special attention should be paid attention to the insulating performance of insulating oil used in converter transformers. In presence of particle and DC component, the insulation design and maintenance should be differentiated compared 
to that in traditional transformers to ensure the insulating performance of insulating liquids. The electric field allowance for the insulation should be different from that of insulation used in conventional AC transformers and the particle limit for insulating oil used in converter transformers should be strictly controlled in case of the electric filed distortion induced by particle which may further cause breakdown of insulation.

\section{Conclusions}

This paper investigated the copper particle effect on the breakdown strength of insulating oil at combined AC and DC voltage. The main conclusions are summarized as follows.

1) The breakdown strength of insulating oil contaminated with copper particles reduces with the increase of the copper particle concentration. There is a liner relationship between the breakdown voltage and logarithm of the copper particle concentration.

2) The experimental and simulation results show that the copper particle will distort the electric field in insulating oil and the uniformity coefficient of electric field will increase, which will increase the breakdown probability of insulating oil and lead to the decline of breakdown voltage.

3) The DC component in applied voltage has significant influence on the breakdown voltage of contaminated insulating oil. The simulation results show that the copper particle collides with the electrode more frequently with more DC voltage component contained in applied voltage, which brings about more times of discharges and decrease the breakdown voltage. For insulating oil used in converter transformers which withstands combined AC and DC voltage, special attention should be paid to its insulating performance.

\section{Appendix}

This appendix illustrates the calculation method of particle number estimation of copper particle with respect to its mass weight.

Assume that the diameter of copper particles of different numbers obey the same diameter distribution illustrated in Fig. 3 and the copper particles are all spheres and uniformly distributed. The copper particles can be classified into $i$ categories by means of their diameters. Define $V$ as the overall volume of copper particles in all diameter ranges and $N$ as the number of all copper particles. The upper and lower boundary of each diameter range are defined as $r_{i \max }$ and $r_{i \min }$, respectively. $r_{i}$ is the average diameter of the $i$ th diameter range and it can be calculated by the following equation

$$
r_{i}=\left(r_{i \max }+r_{i \min }\right) / 2
$$

According to the size distribution of copper particle, volume proportion of particles in each diameter range can be calculated, defined as $\alpha_{i}$. Define $N_{i}$ as the number of corresponding particles in each diameter range and $V_{i}$ as the particle volume of particles in each diameter range. Then the following relationships are obtained.

$$
\begin{gathered}
V_{i}=V \alpha_{i}=4 / 3 \pi r_{i}^{3} N_{i} \\
\sum V_{i}=V \\
\sum N_{i}=N
\end{gathered}
$$

Define $\rho$ as the density of copper particle, the mass weight of particles in each diameter range, defined as $M_{i}$, can be calculated with Eq. (A5).

$$
M_{i}=\rho V_{i}
$$

Define $r_{\text {eq }}$ as the equivalent diameter of all the copper particles, which can be calculated by Eq. (A6).

$$
4 / 3 \pi r_{e p}^{3} N \rho=\sum M_{i}
$$

According to Eq. (A1)-(A6), the relationship between the number of copper particles and the mass weight can be obtained by Eq. (A7).

$$
M=4 / 3 \pi r_{e p}{ }^{3} \rho N
$$

Therefore, the particle number $N$ can be estimated and controlled with the mass weight of the copper particles $M$.

\section{References}

[1] Lu Weixing and B. T. Ooi, "Optimal Acquisition and Aggregation of Offshore Wind power by Multiterminal Voltage-source HVDC," IEEE Transactions on Power Delivery, vol. 18, no. 1, pp. 201-206, Jan, 2003.

[2] Wang X. and Wang Z D, "Particle Effect on Breakdown Voltage of Mineral and Ester Based Transformer Oils," IEEE Conference on Electrical Insulation and Dielectric Phenomena (CEIDP), Quebec, Canada, Oct, 2008, pp. 598-602.

[3] Rain P. and R Tobazã@On, "Behaviour of Free Conducting Particles and Their Role on Breakdown in Oils under AC and DC Voltages," Dielectric Materials, Measurements and Applications, Sixth International Conference on IET, Manchester, England, Sep, 1992, pp. 96-99.

[4] Mahmud S, Chen G and Golosnoy I O, "Experi- 
mental Studies of Influence of DC and AC Electric Fields on Bridging in Contaminated Transformer oil," IEEE Transactions on Dielectrics \& Electrical Insulation, vol. 22, no. 1, pp. 152-160, Feb, 2015.

[5] Samat J. and D. Lacaze, "Micro-particles in Transformer Oil and Dielectric Withstand Effects," Alsthom Review, vol. 11, pp. 47-57, 1988.

[6] CIGRE Working Group 17 of Study Committe 12, "Effect of particles on transformer dielectric strength," CIGRE, 2000.

[7] F. Carraz, P. Rain and R. Tobazeon, "Particle Initiated Breakdown in Quasi Uniform Field in Transformer Oil," IEEE Transactions on Dielectrics \& Electrical Insulation, vol. 2, no. 6, pp. 1052-1063, Dec, 1995.

[8] M. Krins, H. Borsi and E. Gockenbach, "Influence of Carbon Particles on the Breakdown and Partial Discharge Inception Voltage of Aged Mineral Based Transformer Oil," International Conference on Dielectric Materials, Measurements and Applications, IET, Bath, U.K., Sep, 1996, pp. 251-254.

[9] M. Krins, H. Borsi and E. Gockenbach, "Influence of Carbon Particles on the Breakdown Voltage of Transformer Oil," International Conference on Conduction and Breakdown in Dielectric Liquids, Roma, Italy, Jul, 1996, pp. 296-299.

[10] Tobazeon R., "Charges and Discharges of Conducting Particles in a Plane Oil Gap Subjected to a DC Voltage," International Conference on Conduction and Breakdown in Dielectric Liquids, Roma, Italy, Jul, 1996, pp. 309-312.

[11] Mahmud S, Chen G and Golosnoy I O, "Bridging in Contaminated Transformer Oil under DC and AC Electric Field," Journal of Physics: Conference Series, vol. 472, no. 1, pp. 012007, IOP Publishing, 2013.

[12] Wang X, Wang Z D and Noakhes J, "Motion of Conductive Particles and the Effect on AC Breakdown Strengths of Esters," IEEE International Conference on Dielectric Liquids (ICDL), Trondheim, Norway, Jun, 2011, pp. 1-4.

[13] $\mathrm{Li} \mathrm{J,} \mathrm{Hu} \mathrm{Q} \mathrm{and} \mathrm{Zhao} \mathrm{X,} \mathrm{"Partial-discharge}$ Characteristics of Free Spherical Conducting Particles under AC Condition in Transformer Oils," IEEE Transactions on Power Delivery, vol. 26, no. 2, pp. 538-546, Apr, 2011.

[14] Tianyan Jiang, A. Cavallini, G. C. Montanari and Jian Li, "Partial Discharge Activities of Pressboard/oil Insulation under AC plus DC Voltages," IEEE International Conference on Solid Dielectrics, Bologna, Italy, Jun-Jul, 2013, pp. 267-270.

[15] ASTM D877, "Standard Test Method for Dielectric Breakdown Voltage of Insulating Liquids Using Disk Electrodes," 2013.

[16] Martin, Daniel and Wang Z D, "Statistical Analysis of the AC Breakdown Voltages of Ester Based Transformer Oils," IEEE Transactions on Dielectrics and Electrical Insulation, vol. 15, no. 4, pp. 1044-
1050, Aug, 2008.

[17] Ding H Z, Wang Z D and Jarman P, "Dielectric Strength of Aged Transformer Oil Experimental Studies and Statistical Analysis of Breakdown Voltage," Proceeding of the 14th International Symposium on High Voltage Engineering, china, Aug, 2005, pp. 1-5.

[18] Birlasekaran S, "The Measurement of Charge on Single Particles in Transformer Oil," IEEE transactions on electrical insulation, vol. 26, no. 6, pp. 1094-1103, Dec, 1991.

[19] Lebedev N. N. and I. P. Skalskaya, "Force Acting on a Conducting Sphere in Field of a Parallel Plate Condenser," Soviet Physics-Technical Physics, vol. 7, no. 3, pp. 268, Jan, 1962.

[20] Zhou Yuanxiang, Sha Yanchao and Nie Dexin, "Partial Discharge Initiating Process of Transformer Oil under Combined AC and DC Voltage," High Voltage Apparatus, vol. 38, no. 5, pp. 1163-1171, May, 2012.

[21] Choi C, Yatsuzuka K and Asano K, "The Behavior of Spherical Particle under Uniform Electric Field in Silicone Oil," IEEE Conference on Electrical Insulation and Dielectric Phenomena, Victoria, Canada, Oct, 2000, pp. 77-80.

[22] Asano K, Choi C and Kamiya M, "The Behavior of a Spherical Particle under Non-uniform Electric Field in Silicone Oil," IEEE Conference on Electrical Insulation and Dielectric Phenomena, Victoria, Canada, Oct, 2000, pp. 73-76.

[23] Birlasekaran S and Darveniza M, "Micro-discharges from Particles in Transformer Oil," IEEE Transactions on Electrical Insulation, vol. 4, no. EI-11, pp. 162-163, Dec, 1976.

[24] Sharbaugh A. H., J. C. Devins and S. J. Rzad, "Progress in the Field of Electric Breakdown in Dielectric Liquids," IEEE Transactions on Electrical Insulation, vol. 4, pp. 249-276, Aug, 1978.

[25] Mahmud S, Chen G and Golosnoy I O, "Bridging Phenomenon in Contaminated Transformer Oil," International Conference on Condition Monitoring and Diagnosis, Bali, Indonesia, Sep, 2012, pp. 180183.

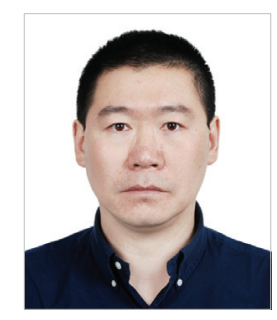

You-Yuan Wang He received the M.S. and $\mathrm{Ph} . \mathrm{D}$. degrees in electrical engineering in 2003 and 2008, respectively from Chongqing University, Chongqing, China. He is currently a professor of High Voltage and Insulation Technology Department at Chongqing University. His major research interests include dielectric properties and space charge behavior of nanocomposites, online detection of insulation condition in 
electrical devices, and insulation fault diagnosis for high voltage equipment. He is an author and coauthor of more than 50 journal papers and 30 papers published in proceedings of international conferences.

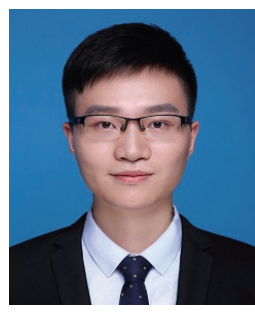

Yuan-Long Li He received the B.E. degree in electrical engineering and automation in 2014 from Chongqing University, China. He is currently a master candidate at School of Electrical Engineering, Chongqing University. His research interests include insulation fault diagnosis of electrical equipment, thermal and mechanical phenomena in discharges of transformer oil.

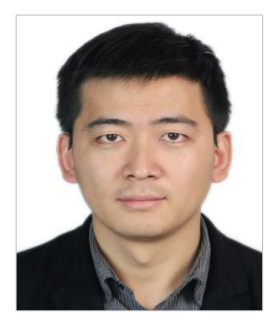

Chao Wei He was born in Shandong, China in 1984. He received the master degree in electrical engineering and automation in 2010 from Chongqing University, China. Now he works in Jiangsu Electric Power Company Research Institute of China and engaged in equipment condition assessment and diagnosis technology of electrical equipment.

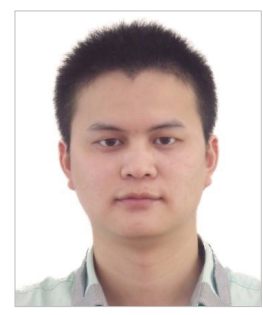

Jing Zhang He received the B.E. degree in electrical engineering and automation in 2012, from China University of Mining and Technology, Xuzhou, China. He is currently a Ph.D. candidate at School of Electrical Engineering, Chongqing University. His major research interests include condition monitoring and insulation fault diagnosis of electrical equipment, especially aging and diagnosis for power transformer insulation.

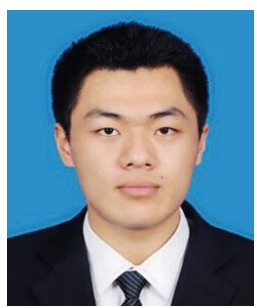

$\mathbf{X i ~ L i ~ H e ~ w a s ~ b o r n ~ i n ~ J i a n g s u , ~ C h i n a ~ i n ~}$ 1993. He received the B.E. degree in electrical engineering and automation in 2015 from Chongqing University, China. He is currently a master candidate at School of Electrical Engineering, Chongqing University. His major research interests is diagnosis for power transformer insulation. 